\title{
FEASIBILITY EVALUATION AND CRITICAL FACTOR ANALYSIS FOR SUBWAY SCHEDULING
}

\author{
T. ZHU, J.M. MERA, E. CASTELLOTE \& J. LÓPEZ \\ Railway Technology Research Centre, Polytechnic University of Madrid, Spain.
}

\begin{abstract}
In strategic subway scheduling stage, the conflict sometimes comes from different requirements of the subway operator. This study aims to investigate the significant factors concerning strategic subway scheduling problem and to develop an automatic procedure of feasibility analysis in subway scheduling. To this end, accurate simulation of train movement (via a simulator, named HAMLET) is applied first by considering the line geography, train performances, actual speed restrictions, etc. The critical elements of subway scheduling and their correlations are then studied and a bound structure of the critical factors is established. The feasibility of primary plan requirements is analysed with the restrictions of the bound structure. Infeasible aspects and possible adjustments are shortly discussed. Finally, the subsequent applications including schedule generation and optimization according to various objectives are indicated as well.

Keywords: factor analysis, feasibility evaluation, periodic scheduling, subway, train scheduling.
\end{abstract}

\section{INTRODUCTION}

There are several distinctive features of subway schedule:

- Subway schedules are remarkably periodic. And the period of a subway schedule is actually short, typically ranging from tens of minutes to several hours. Thus, for both a train and a station the daily schedule they follow consists of multiple periods. Each period of the schedule for a train is called a cycle of the train.

- The service of subway is required to be highly frequent. In other words, the intervals between trains are quite short.

- Within a certain travel zone, the satisfaction of passengers does not critically rely on the punctualities of all trains in the timetable (except the first and last trains on schedule), but the average waiting time and total travel time.

- Only a few stations are equipped with siding tracks (for trains in failure); i.e. it is difficult to prevent the impact of some local failure to propagate into a global scope.

- Possible conflicts come from multi-objective requirements of the subway operator.

There are a wide variety of elements involved in railway scheduling. As in other kinds of scheduling problems, over a time, periodic event scheduling problem (PESP) has played a dominant role in railway scheduling problem by considering the basic aspects, including the arrival and departure times of trains at each station and the assignment of train routes based on the infrastructure resource. For example, as an early study to exploit the PESP to model and construct railway timetables, Odijk [1] first built a visual graph to express the timetable structure where a vertex represents an event and an arc represents a constraint. 
The scheduling problem is solved by searching a feasible and good timetable in this constraint graph. Similarly Liebchen et al. [2] also modelled a periodic timetable optimization task as a PESP. Besides, it takes the amount of rolling stock required, average passenger changing time (connection time), average speed of lines, etc., into account.

Besides the reasonable travel times and waiting times, other interests have also been concerned. In the scheduling and rescheduling model proposed by [3], the total final delay and the cost associated with delays are considered. Miyatake et al. [4] investigated the relation between running time and the energy consumption in the whole section inter-stations and further proposed an adjustment law to minimize the total energy consumption by optimizing all running times. In this study the dwell time at each station is unchanged. In contrast, in order to optimize the utilization of regenerated energy in train braking stage, Yang et al. [5] proposed a cooperative scheduling approach, which allows the dwell times to vary. It particularly considers the successive trains and allows the regenerated energy from the braking train to be used by the accelerating train simultaneously. In the earlier model, the energy is the primary factor being taken into consideration. Still in their subsequent work, an extension version using two-objective model was proposed where the energy saving is maximized and the passenger waiting time is minimized [6].

To sum up, in subway train scheduling, the main concerns are arrival times, stop and waiting time at each station, passenger travel times, delays and energy consumption, etc. However, the minimum train intervals, train turning time at terminals and required number of trains are not widely discussed. Instead, these elements are supposed to be a set of static and known parameters in most subway scheduling problems. Thus, this paper studies these elements first at a more fundamental layer, integrating the physical properties of line and the comprehensive behaviours of train. Then it aims to develop an automatic procedure of feasibility analysis in subway scheduling based on the investigation of the key factors in periodic subway scheduling problem. Furthermore, some experience rules to solve the infeasibility are shortly discussed in a demonstrative instance.

\section{CRITICAL FACTORS OF SUBWAY SCHEDULING}

\subsection{Accurate simulation of train movement}

First, basic technical factors for accurate simulation of train movement are analysed to study the actual information required in real world.

A simulation tool named HAMLET (Herramienta Avanzada Multidisciplinar para Líneas Eléctricas de Trenes) is applied to reconstruct the infrastructure and signalling system and simulate the train behaviours. As described in the previous work of HAMLET [7], aspects of infrastructure, train, and electrical and signalling systems are all considered (Fig. 1).

There are two classes of infrastructure data of line. The static configurations include positions of key point, lengths, slopes, curves, tunnels and bridges, civil speed restrictions, segment operational speed limitation, feasible route table, etc. And the dynamic information here refers to the track description, which mainly depends on the signalling system and train movement on the lines.

Signalling and electrical systems represent the most important trackside support systems. They are integrally designed to satisfy the applications of train control system.

Train data mainly depend on the train type (locomotive and car specifications, the composition of train, weight, length, rated number of passengers, electrical and mechanical 


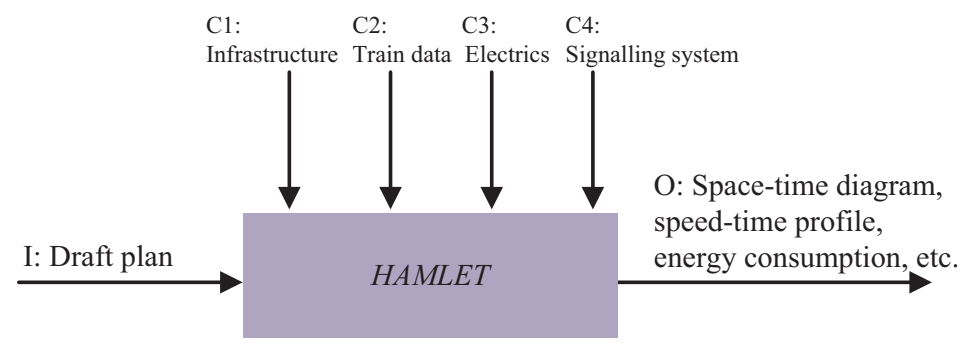

Figure 1: System chart of HAMLET.

parameters, etc.). The traction and braking curves are important references in the accurate simulation of a train. It is noted, however, that each train is considered to be as a whole and its specific performances are represented in the actual speed profile and energy consumption profile.

Actually, the input of HAMLET can be a route description for a single train as well as a draft plan of multi-trains according to the user requirements. When it is a route description for a particular train type, the HAMLET outputs the simulation results of this type of train based on the given routes, such as the running time on each piece of section, actual speed curves, driving modes, energy estimations, etc. Besides, actions such as changing driving mode or using the temperate speed limitations can still be added to a draft plan, and correspondingly it leads to a distinct set of results of the train. Multi-train simulation is based on each single train performance, required plan (input), and track description and the control levels. Its outcomes may indicate the feasibility or even potential conflicts of the draft plan.

\subsection{Varied requirements}

In many cases the uniform interval $I_{0}$ between given trains is required by the subway operator once the information of line facility, signalling system and train types are known. The total number of needed trains $N$ is the variable to be determined.

On the contrary, in some other cases, the feasible interval $I$ is questioned whilst the total number of available trains $N_{0}$ (and the composition of each train) is provided as a precondition.

Besides, the average stop and waiting time at stations $W_{0}$ and the average time for changing train direction at terminals $C_{0}$ are usually given. Nonetheless, some of the waiting times $W$ and direction turning around times $C$ in actual scheduling can be determined in any situation.

\subsection{Fundamental factors}

Other critical factors considered to analyse and optimize the operating plans are expressed as follows:

- In case there is more than one type of trains, synthetic train speed index $\overline{v p}$ of train type $i$ is defined. It is a relative value that is calculated by

$$
\overline{v p}_{i}=\frac{V_{i}}{V_{0}},
$$


where $V_{0}$ is the mean velocity of the referred train type and $V_{i}$ is the average speed of train type $i$. As the trains for a line are known, $\overline{v p}_{i}$ of each train type is determined. The index $\overline{v p}_{i}$ values 1 if there is only a unique train type used in a line.

- Minimum operation times: waiting time at station $W_{\min }$, time to switch on the locomotive of the $\operatorname{train} C_{\min }$, i.e. direction turning time for a train.

- Minimum intervals $I_{\min }$ depend on the most restrictive conditions of key points, such as the limited speed and the additional operation time of a switch, etc. Thus, it should be a key point to obtain its minimum interval. As there usually are a set of key points on a line, $I_{\min }$ is a vector. The finally adopted interval $I$ of the general schedule is not smaller than the maximum value of $I_{\min }$.

- Route decision $r: r$ is the selected route set from the route table. If a route is selected, correspondingly the running time set $B_{r}$ of all segments of the route (related to complement time and required interval and switch positions) is also referred.

\subsection{Integral effect}

There are some variables that can be only integers. A frequent one is the total number of trains planned to use in a particular line, i.e. $N$ is an integer.

As the periodic characteristic of subway schedule, the second cycle of the train that departs first has to be scheduled with the same interval $I$ from the train that departs last; commonly an additional time has to be introduced to complement the difference between the schedule period and the single cycling time of train. This complement time $t_{\text {comp }}$ is expressed by

$$
t_{\text {comp }}=T-t_{c y c},
$$

where $t_{c y c}$ is the total travel time of a particular train type to complete a cycle in a condition that the train path (a set of routes) and the basic time for waiting and for changing train direction are given. $T$ is the schedule period.

$$
T=N I
$$

The complement time sometimes can be a flexible buffer for slight delays. Nevertheless, it might also cause potential conflicts at terminals since in many cases the complement time is distributed to the train dwelling time or turning time at terminals and it increases the time to complete the usage of the platforms for a train (the trains usually are not planned to enter the depot).

\section{FACTOR CORRELATIONS}

The critical factors can be further categorized into technical and decidable groups. As the train data are given, the minimum operation times $W_{\min }$ and $C_{\min }$ and the train speed index are determined. Taking the line data and signalling system into account, the interval restrictions $I_{\min }$ are technically definite, yet they are to be measured. By contrast, the waiting times, direction turning around times, intervals in service, total number of trains and even the train path are decidable, either by operator's expectations or other objectives. Figure 2 shows the logical associations of these factors.

To determine the variables in the latter group, the correlations of factors are further studied by statistical analysis. Correlation matrix of factors is shown in Table 1 where each row 
Factors and intermediate variables

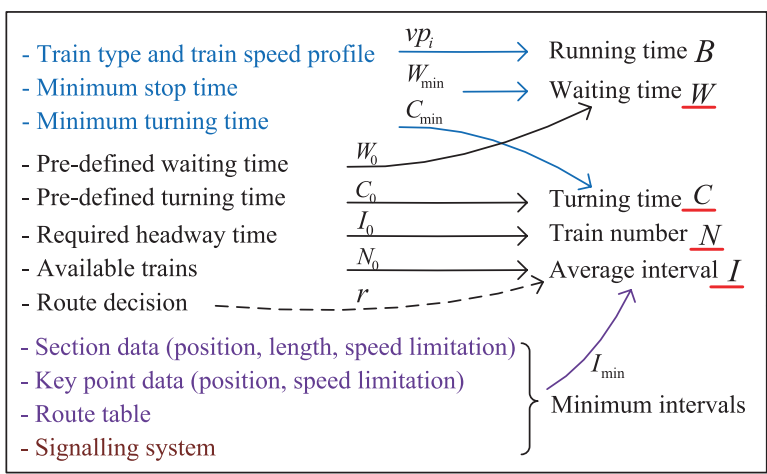

Objectives

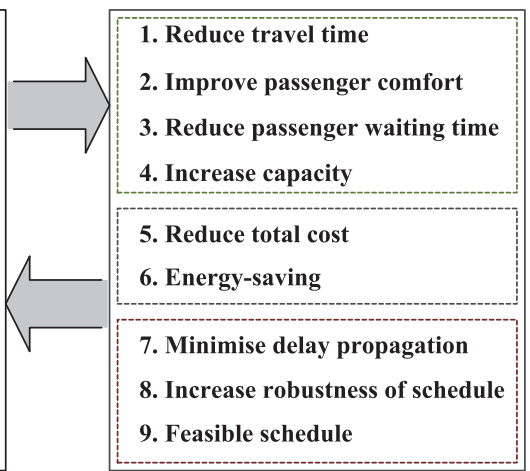

Figure 2: The principal associations of factors.

implies how a decision variable depends on other factors and the bigger a correlation value indicates the more related that pair of factors. Some important correlations (bold values) are coincident with the common rules we obtain. First we note that between two order stops of train only the strictest key point is denoted by the index $K$. The frontal dwelling time (in the train movement direction) of key point $K$ is denoted by $C_{k}$ or $W_{k}$. Thus, some important correlations showed in Table 1 can be partly explained as follows:

- The minimum interval of a key point depends on the position and the type of train stop (to keep or to change the direction of train); besides, it is related to the average speed to travel through the key point. They are expressed, respectively, by

$$
\begin{aligned}
& I_{\text {min }}(k)=a W_{k}+b \overline{v p}_{i}+c, \\
& I_{\text {min }}(k)=d C_{k}+e \overline{v p}_{i}+f,
\end{aligned}
$$

where $a, b, c, d, e, f$ are the parameters of a given system.

- The final determined interval should not be smaller than the minimum allowed interval of any key point; that is,

$$
I \geq \max \left\{I_{\min }\right\}
$$

If the number of trains and the train performances are given, then

or

$$
\begin{gathered}
I \geq t_{c y c} / N_{0}, \\
I=\left(t_{c y c}+t_{c o m p}\right) / N_{0} .
\end{gathered}
$$

- In a contrary case the interval in service is known, and the number of trains satisfies

$$
N=\left(t_{c y c}+t_{\text {comp }}\right) / I_{0} .
$$


Table 1: Correlation matrix of factors in statistical analysis.

\begin{tabular}{lcccccccccc}
\hline & Pos $^{*}$ & $I_{\min }$ & $C_{k}$. & $W_{k}$ & $V_{i}$ & $I_{0}$ & $N_{0}$ & $t_{c y c}$ & $t_{c o m p}$ & $r$. \\
\hline Pos & 1.000 & -0.387 & -0.322 & 0.294 & 0.320 & 0.021 & -0.028 & -0.029 & -0.004 & -0.003 \\
$I_{\min }$ & $-\mathbf{0 . 3 8 7}$ & 1.000 & $\mathbf{0 . 4 0 1}$ & $-\mathbf{0 . 5 3 0}$ & $-\mathbf{0 . 6 7 0}$ & 0.213 & -0.184 & -0.043 & -0.028 & -0.079 \\
$C$ & -0.322 & 0.401 & 1.000 & -0.8 & & & -0.049 & -0.021 & -0.072 & -0.074 \\
$W$ & 0.294 & -0.530 & -0.831 & 1.000 & 0.654 & -0.006 & 0.014 & 0.019 & 0.006 & 0.038 \\
$V_{i}$ & 0.320 & -0.670 & -0.499 & 0.654 & 1.000 & 0.052 & -0.132 & -0.209 & -0.084 & -0.139 \\
$I$ & 0.021 & $\mathbf{0 . 2 1 3}$ & 0.033 & -0.006 & 0.052 & 1.000 & $-\mathbf{0 . 8 9 0}$ & $-\mathbf{0 . 2 2 6}$ & -0.127 & $-\mathbf{0 . 4 8 7}$ \\
$N$ & -0.028 & -0.184 & -0.049 & 0.014 & -0.132 & $-\mathbf{0 . 8 9 0}$ & 1.000 & $\mathbf{0 . 6 0 5}$ & $\mathbf{0 . 3 6 5}$ & $\mathbf{0 . 7 0 1}$ \\
$t_{\text {cyc }}$ & -0.029 & -0.043 & -0.021 & 0.019 & -0.209 & $-\mathbf{0 . 2 2 6}$ & $\mathbf{0 . 6 0 5}$ & 1.000 & $\mathbf{0 . 3 2 5}$ & $\mathbf{0 . 7 7 9}$ \\
$t_{\text {comp }}$ & -0.004 & -0.028 & -0.072 & 0.006 & -0.084 & $\mathbf{- 0 . 1 2 7}$ & $\mathbf{0 . 3 6 5}$ & $\mathbf{0 . 3 2 5}$ & 1.000 & 0.092 \\
$r$. & -0.003 & -0.079 & -0.074 & 0.038 & $-\mathbf{0 . 1 3 9}$ & $-\mathbf{0 . 4 8 7}$ & $\mathbf{0 . 7 0 1}$ & $\mathbf{0 . 7 7 9}$ & 0.092 & 1.000 \\
\hline
\end{tabular}

"Position of the key point on the line.

- When there are many types of trains to run on the line, the train type and its speed index are one of the influencing factors of the single cycling time of the train.

$$
t_{c y c}=h \overline{v p}_{i}+l
$$

- Route decision also has an effect on the train cycling time and the variable pair: the adopted number of trains $N$ and interval $I$.

- Last but not least, the complement time has to be distributed to the dwelling times and it satisfies eqns (10) and (11).

$$
\begin{aligned}
& t_{\text {comp }}= \sum\left(W_{k}-W_{0}\right)+\sum\left(C_{k}-C_{0}\right), \\
& t_{\text {comp }}<\sum\left(I-I_{\text {min }}(k)\right),
\end{aligned}
$$

$W_{0}$ and $C_{0}$ are pre-defined and $W_{0}, W_{k} \geq W_{\min }, C_{0}, C_{k} \geq C_{\min }$.

\section{PLANNING FEASIBILITY}

The difficulty to estimate the feasibility of the user requirements lies on the correlative variation of the general restriction structure. In other words, all the restrictions are not constant. Fortunately, most factors can be related to the key factor, minimum interval vector $I_{\min }$. To sum up, the procedure of feasibility evaluation may include the following steps:

(s1) Calculate $I_{\min }$.

(s2) Obtain $t_{c y c}$ (on the basis of $W_{k}=W_{0}$ and $C_{k}=C_{0}$ ). 


\section{COMPRAIL}

(s3) Obtain the coefficients in eqns (4), (5) and (9).

(s4) Check the feasibility by inequalities in eqns (6), (7a), (7b), (8) and (11).

After step (s3), linear bounds of each desired variable may be visualized in a bound diagram; e.g. Fig. 3 shows a bound diagram of acceptable interval of a line where the train performance and train path are definitive.

Step (s4) deduces a conclusion by verifying all bounds of the variables. It results in a positive outcome when all requirements can be satisfied within the bounded domain. Otherwise, it identifies that the user requirements of plan are infeasible. The user may obtain inspired information to adjust the requirements.

Take the line given in Fig. 4 as an example. 21 key points of the line are taken into account in $I_{\min }$ investigation, among which $1,3,5$ and 14 are the key points that can be related to the times to change train direction, i.e. $C_{k}$; others are related to the waiting times $W_{k}$. The available number of trains of certain type is known, $N_{0}=22$. It is queried whether it is feasible to schedule the trains at an interval of $100 \mathrm{~s}$.

When the average speeds of train types are not obviously different $\left(\overline{v p}_{i} \in\{1,0.881\}\right.$ in this example), some parameters appear as Table 2 shows. In detecting $I_{\min }$ the initial setting

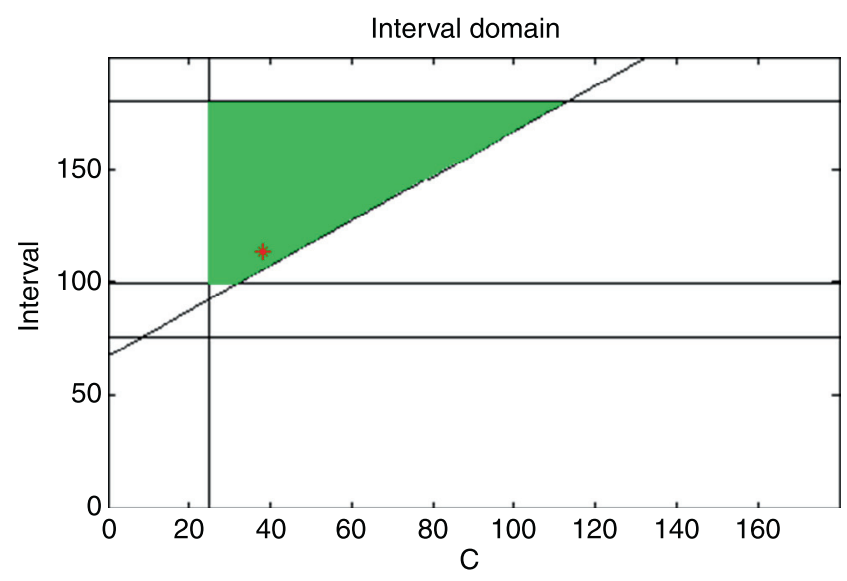

Figure 3: An instance of linear bounds of the interval (along a given train path).

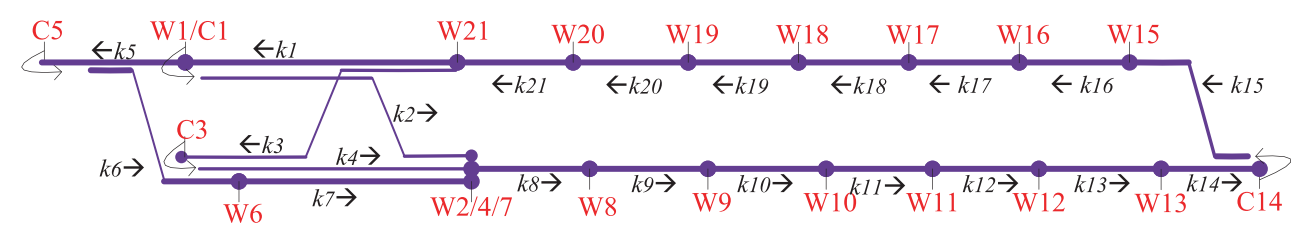

Key points and times in Route set 1: $k 1-\mathrm{C} 1-k 2-\mathrm{W} 2-k 8-\mathrm{W} 8-k 9-W 9-\ldots-k 14-\mathrm{C} 14-k 15-\mathrm{W} 15-k 16-\mathrm{W} 16-\ldots-k 21-\mathrm{W} 21-k 1-\ldots$

Key points and times in Route set 2: k3-C3-k4-W4-k8-W8-k9-W9-...-k14-C14-k15-W15-k16-W16-...-k21-W21-k1-...

Key points and times in Route set 3: k1-W1-k5-C5-k6-W6-k7-W7-k8-W8-...-k14-C14-k15-W15-k16-W16-...-k21-W21-k1-...

Figure 4: A subway line with 7 stations and trains can only change the direction and track at terminal stations; 3 train paths with corresponding key points and routes to be selected. 
includes $W_{0}=30, C_{0}=30$, and $\overline{v p}_{i}=1$. Table 3 shows the results of feasibility estimation varying from train path decision.

From the results we can conclude that the requirement of running trains at an interval of $100 \mathrm{~s}$ in the given case is possible. It is infeasible only when there is no route set satisfying the requirement and restrictions.

Table 2: Parameters of correlations and measured minimum intervals of partial key points.

\begin{tabular}{lccccc}
$a, b, c, d, e, f, h, l$ & $I_{\min -} C_{1}$. & $I_{\min -} C_{3}$ & $I_{\min -} C_{5}$ & $I_{\min -} C_{14}$ & $\max \left\{I_{\min -} W\right\}$ \\
$1,0,45,1,-42,112,-866,2939$ & 115 & 106 & 100 & 99 & 75 \\
\hline
\end{tabular}

Table 3: Feasibility estimation by route set.

\begin{tabular}{lccccccc}
\hline Route set $r$ & $t_{c y c}$ & $I$ domain & $I$ & $N$. & $\sum\left(I-I_{\min }(k)\right)$ & Feasibility \\
\hline 1 & 1911 & {$[115, \hat{I}]^{*}$} & 100 & - & - & - & No \\
2 & 1910 & {$[106, \hat{I}]$} & 100 & - & - & - & No \\
3 & 2053 & {$[100, \hat{I}]$} & 100 & 21 & 47 & 401 & Yes \\
$1 \& 2^{* *}$ & 1971 & {$\left[75, C_{1}+15\right]$} & 100 & 20 & & $321+C_{1}$ & Yes \\
\hline
\end{tabular}

" $\hat{I}$ is the maximum value of the acceptable interval, given by the user.

${ }^{* * *} 1 \& 2$ means successive trains alternately take the route set 1 and route set 2 .
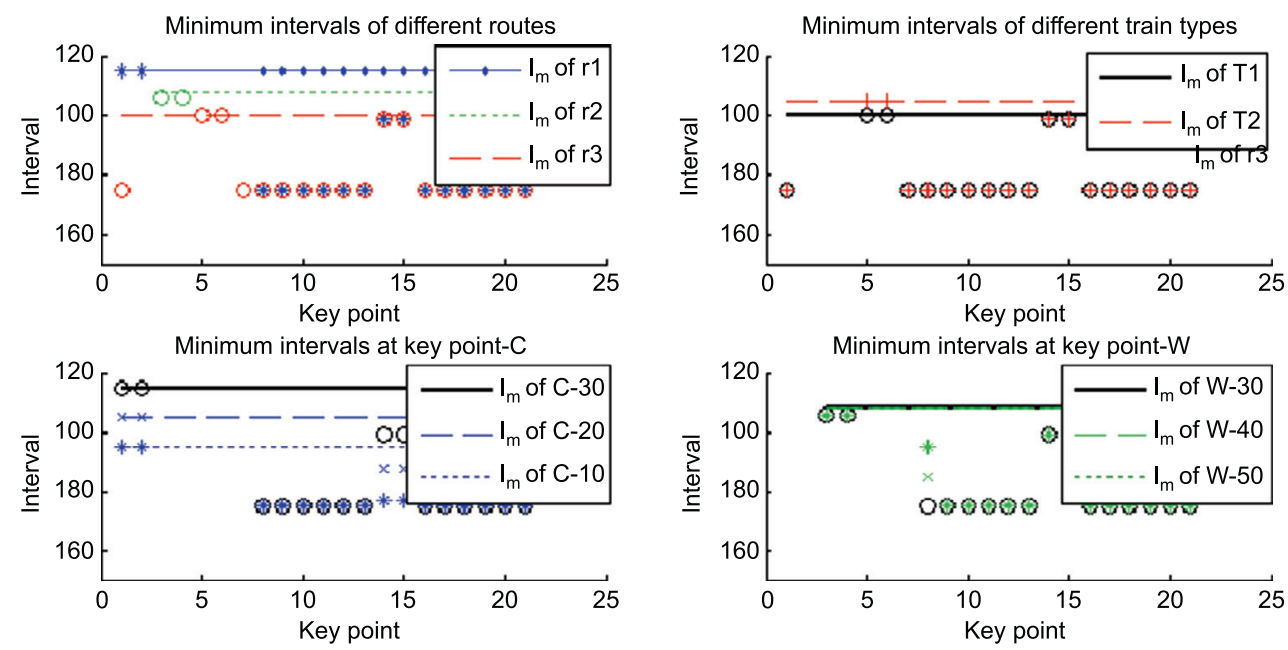

Figure 5: Distribution information of the intermediate variable $I_{\min }$. 


\section{COMPRAIL}

Besides further information of the minimum interval values we obtained by setting different routes, train types or distributing different dwelling times are illustrated in Fig. 5, from which two experience rules are summed up.

- \#1: Only the key point whose $I_{\min }(k)$ equals $\max \left(I_{\min }\right)$ of certain route set directly affects the acceptable interval $I$ of the route set;

- \#2: The acceptable interval $I$ of a regular periodic schedule depends on $I_{\min }$ of the lowerspeed train type.

Thus, the user may decide whether to adjust the dwelling time related to this kind of key point in order to obtain an appropriate interval $I$. But certainly, other variables and restrictions may be re-analysed as well.

\section{APPLICATIONS}

As shown in Fig. 6, further applications of feasibility evaluation include plan generation and optimization. Actually, only when the general feasibility is verified, it comes to have practical significance to apply algorithms and methods to generate and optimize the plan.

To generate a feasible plan, we only have to follow two more steps after the feasibility evaluation steps (s1 to s4):

(s5) Determine the favourable route set $r$ and corresponding $t_{\text {comp }}$.

(s6) Determine the feasible $C$ and $W$ by distributing $t_{\text {comp }}$.

Here we consider the data of the train type(s) are pre-defined.

To optimize the plan, objectives are to be clarified first. Some of the objectives concluded in Fig. 2 can only be achieved by combining with the train control (e.g. passenger comfort), and some can be approached by adding objective functions to the scheduling model. For example, functions (12) and (13) are, respectively, defined to minimize the total travel time and to maximize the robustness of the plan.

$$
\begin{array}{lr}
\text { Minimise: } & B_{r}+\sum(W(k)) \\
\text { Maximise: } & \min \left(I-I_{\min }(k)\right)
\end{array}
$$

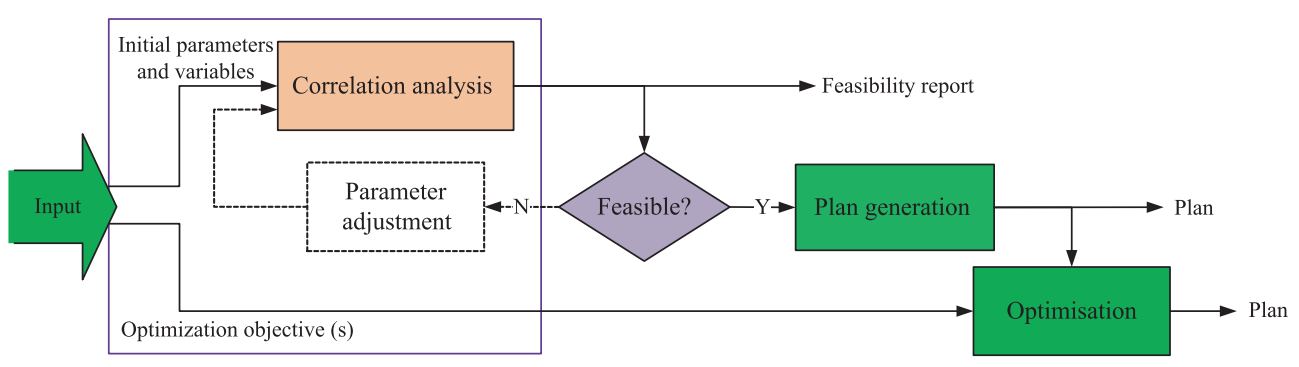

Figure 6: Possible applications based on feasibility analysis. 


\section{CONCLUSIONS}

Actually, there are a number of factors to be concerned in practical subway scheduling process. These factors are quite basic and detailed but sometimes difficult to obtain for their variability and correlations. In many subway scheduling models, detailed factors are not considered and constant parameters are commonly used to simplify the problem. In this research, we investigated the main factors which might be considered in periodic subway scheduling problem from a more fundamental level.

Based on the correlation study of the critical factors, we established a set of internal restrictions among the factors, namely bound structure. Via this dynamic bound structure we realized the feasibility evaluation of the original requirements proposed by the subway operator (the user). Subsequently, the subway schedule (plan) can be generated if the user requirements are practicable and further optimized according to particular objectives. Otherwise, infeasible aspects are analysed, and possible adjustments which might produce a feasible plan can be proposed according to the available resources of the system.

Feasibility analysis is an indispensable part of work in practical subway scheduling, and more complex situations (such as multi-train type, more shared conjunctions on different routes, etc.) are to be included in the research.

\section{REFERENCES}

[1] Odijk, M.A., A constraint generation algorithm for the construction of periodic railway timetable. Transportation Research Part B: Methodological, 30, pp. 455-464, 1996. DOI: 10.1016/0191-2615(96)00005-7.

[2] Liebchen, C. \& Möhring, R. H., A case study in periodic timetabling. Electronic Notes in Theoretical Computer Science, 66, pp. 18-31, 2002. DOI: 10.1016/S15710661(04)80526-7.

[3] Törnquist, J. \& Persson, J.A., N-tracked railway traffic re-scheduling during disturbances. Transportation Research Part B: Methodological, 41, pp. 342-362, 2007. DOI: 10.1016/j.trb.2006.06.001.

[4] Miyatake, M., Kuwahara, R. \& Nakasa, S., A simple adjustment of runtimes between stations for saving traction energy by means of mathematical programming, presented at the Computers in Railways XIII, New Forest, UK, 2012.

[5] Yang, X., Li, X., Gao, Z., Wang, H. \& Tang, T., A cooperative scheduling model for timetable optimization in subway systems. IEEE Transactions on Intelligent Transportation Systems, 14, pp. 438-447, 2013. DOI: 10.1109/TITS.2012.2219620.

[6] Yang, X., Ning, B., Li, X. \& Tang, T., A Two-objective timetable optimization model in sybway systems. IEEE Transactions on Intelligent Transportation Systems, 15, pp. 1913-1921, 2014. DOI: 10.1109/TITS.2014.2303146.

[7] Soler, M., López, J. \& Mera, J.M., Simulation system for the optimization of a block distribution under the ERTMS-1 signalling system, presented at the Computers in Railways XIII, New Forest, UK, 2012. 